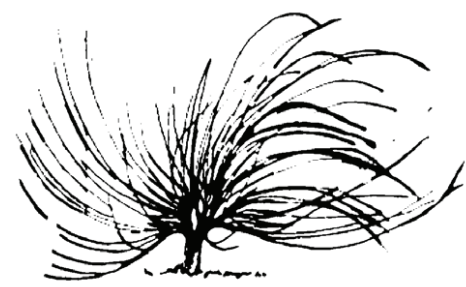

\title{
Relevancia del Uso de las Videoconferencias Como Recurso Didáctico en la Cátedra de Filosofía de la Universidad Estatal a Distancia de Costa Rica
}

\author{
Ricardo Antonio Villalobos Paniagua ${ }^{1}$ \\ Universidad Estatal a Distancia \\ San José, Costa Rica \\ ravp1970@yahoo.com
}

\begin{abstract}
Resumen
Este artículo tiene como meta y fin mostrar, fundamentalmente, de parte del autor los resultados de su investigación llevada a cabo con el propósito de obtener su grado de Magister en Docencia Universitaria de la Universidad Nacional. Este estudio tuvo como propósito conocer y valorar el papel de un medio audiovisual altamente relevante y pertinente en la educación a distancia, a saber: la videoconferencia, y en un espacio académico particular de, y en, la Universidad Estatal a Distancia de Costa Rica (UNED): la Cátedra de Filosofía que pertenece a la Escuela de Estudios Generales.
\end{abstract}

Recibido: 5 de marzo de 2014 - Aprobado: 18 de agosto de 2014

1 El autor es docente actualmente en la Universidad Estatal a Distancia de Costa Rica (UNED) con las Cátedras de Filosofía y de Métodos de Estudio a Distancia e Investigación; en la Universidad de Costa Rica con la Escuela de Estudios Generales, Sección de Filosofía y Pensamiento; en la Universidad Nacional de Costa Rica con Educología (CIDE) en la Licenciatura en Pedagogía con énfasis en Didáctica, es director de tesis en el Programa de Licenciatura en Docencia de la UNED. Es Bachiller en Filosofía y Humanidades, Licenciado en Filosofía, Licenciado en Ciencias de la Educación con énfasis en Docencia y Magister en Educación con énfasis en Docencia Universitaria por la Universidad Nacional de Costa Rica. 
Palabras clave: educación, videoconferencia, educación a distancia, tecnologías educativas, filosofía

\begin{abstract}
This article aims to show the results of a research project carried out by the author in order to obtain his Master's Degree in University Teaching from "Universidad Nacional." This study intended to know and assess the role of a highly relevant and pertinent audiovisual resource in distance education, namely, video conferencing in a particular academic space, Distance State University of Costa Rica (UNED, for its acronym in Spanish) in the Chair of Philosophy that belongs to the School of the Humanities.
\end{abstract}

Keywords: education, video conferencing, distance education, educational technology, philosophy

\title{
Introducción y justificación de la investigación
}

L a labor investigativa, de la cual este artículo se convierte en un extracto sintético fundamentalmente, se debió a la consideración particular, acerca de la pertinencia de las tecnologías educativas (TIC) en la educación, en general, especialmente, en los sistemas de educación superior, por cuanto sus incidencias e influencias, en los nuevos modos de educar, de enseñar. Es así como se consideró este estudio desde uno de estos "nuevos medios" de la educación: la videoconferencia específicamente, como recurso didáctico y metodológico.

Este estudio se limitó en el espacio y el tiempo, a saber, en la Cátedra de Filosofía de la Universidad Estatal a Distancia (UNED), por ello, se indagó con los docentes tutores, una muestra representativa de estudiantes y especialistas en videoconferencias de la dependencia de la UNED.

El estudio se considera justificado por la relevancia de propiciar discusiones acerca del evidente impacto del uso de las tecnologías educativas, en este caso particular, en la educación a distancia. No se buscó desarrollar un discurso que desprestigiara a la tecnología actual, pero sí considerar la posibilidad de entresacar teorías a partir de los resultados. 
Por ende, se quiso posibilitar otra opción de razonamiento, análisis, confrontación y entendimiento del asunto, porque la videoconferencia en la educación a distancia es de suma vialidad, por tanto, se consideró de un merecido estudio de investigación que incluyese algunas deducciones, incluso éticas.

Además de las motivaciones personales del investigador, la filosofía fue inmiscuida en el problema central del estudio en tanto evento milenario en el campo de la educación, la cual también hoy se encuentra inmersa en el discurso de las llamadas tecnologías educativas

El estudio enriquece la percepción del trabajo de una cátedra de filosofía particular, pero en prospectiva a nuevos estudios en nuestro país, sean en relación con este tema o con otros símiles y del filósofo en cuanto a su participación como docente, su responsabilidad ética para con esas tecnologías educativas y su manifestación y expresión respecto de/en cuanto y tanto las tecnologías educativas favorecen o desfavorecen la enseñanza de la filosofía. La videoconferencia se convierte en un fenómeno educativo oportuno para visualizar las nuevas formas de acción en la educación filosófica del siglo XXI, respecto de sus alcances y aplicaciones didácticas.

La filosofía, por su naturaleza académica, ha de ser considerada como una rama del saber con perspectivas educativas muy distintas a cualquier otra instancia del saber, especialmente por cuanto su necesidad en el contacto personal (socialización) para la vivencia del conocimiento. Parece que la teoría nos lleva a creer que una clase de filosofía es poco viable desde una clase virtual; sin embargo, al indagar en este tema y asunto se podrían lograr nuevas conclusiones que "reinventen" las metodologías para elaborar una clase filosófica. La recomendación es visualizar el documento de la investigación integralmente en la Oficina de la Maestría en Docencia de la Universidad Nacional.

\section{El problema de la investigación y sus objetivos}

El interés de la investigación de entrever y buscar conclusiones viables y reales que muestren cómo solucionar un dilema: El uso y buen uso, o no, de las videoconferencias en la Cátedra de Filosofía de la UNED. Por ello, se planteó el siguiente enunciado que enmarcaba el problema de investigación: 
¿Cuál es la relevancia de la implementación de la video conferencia en la educación a distancia y específicamente en la Cátedra de Filosofía de la Universidad Estatal a Distancia (UNED)?

En tanto, los objetivos se enunciaron de la siguiente manera:

Objetivo general: Valorar la relevancia de las videoconferencias en la educación a distancia en Costa Rica, específicamente, en la UNED y desde la Cátedra de Filosofía.

Los objetivos específicos, consecuentes en la dirección metodológica de la investigación, fueron:

1. Analizar el recurso de la videoconferencia como un medio de aprovechamiento pedagógico en el campo de la filosofía y en la educación superior.

2. Explorar la funcionalidad de la videoconferencia en el campo pedagógico y andragógico universitario.

3. Conocer, desde los diferentes modelos teóricos, las ventajas y desventajas de la implementación de la videoconferencia en el área de la filosofía en la educación superior.

4. Determinar una propuesta pedagógica que posibilite la aprehensión y consecuente valoración de la videoconferencia como medio didáctico entre los estudiantes universitarios de la UNED.

\section{La fundamentación teórica}

Ruiz (2009) reflexiona acerca de ideas teóricas como la siguiente: “(...) queda claro que la educación, en cualquiera de los matices o formas que se presente, constituye el curso de solución más efectivo para alcanzar el desarrollo pleno de las potencialidades de cada ser humano" (p. 72). Así es como la autora hace referencia, de alguna manera, a las modalidades educativas diversas y su papel preponderante en el desarrollo humano.

El modelo pedagógico de la educación a distancia, caso de la investigación en cuestión, es uno de implementación reciente, podríamos decir, y debido al auge de las tecnologías educativas informáticas e instrumentales, que nacen especialmente y a partir de la segunda mitad del 
siglo XX, con la venida de la televisión, la radio y su difusión mundial, la internet, los nuevos medios de comunicación (telefonía fija y celular, periódicos virtuales, e instrumentos como las mecanismos físicos para proyectar imágenes o difundir sonido, etcétera).

Con el advenimiento y auge de la educación a distancia, aparecen nuevas necesidades en el proceso o fenómeno educativo que deben ser solventadas inmediatamente por los teóricos de la educación. La educación a distancia no nace sino a partir de las innovaciones tecnológicas llevadas al proceso educativo.

La educación a distancia establece relaciones entre los actores educativos a partir de situaciones muy particulares, en las cuales se deben considerar, por ejemplo, estudiantes que trabajan, estudiantes que viven en lugares lejanos geográficamente de un centro universitario, tutores que más que profesores tradicionales son docentes itinerantes, docentes de la virtualidad, y otras. Zelaya (2007, pp. 49-50) considera que para desarrollar el modelo de educación a distancia se deben considerar los siguientes elementos:

- Los recursos tecnológicos: satélites, decodificadores, computadoras, acceso a la red, televisores, videocaseteras, teléfono, fax, impresora y paquetes multimedia con sus características, potencialidades y limitaciones.

- $\quad$ Los espacios de aprendizaje, con la incorporación de las TIC se ha diversificado el concepto de "aula", ampliados con las aulas remotas o virtuales y demostrando que el aprendizaje no es patrimonio exclusivo del espacio escolar.

- Los cursos, que requieren de la participación de especialistas, productores y diseñadores y de un coordinador pedagógico y del modelo instruccional para asegurar la unidad de la propuesta, conforme el modelo curricular establecido.

- Los tutores pedagógicos (docentes) encargados de establecer los procesos comunicacionales, que pueden darse como procesos individuales o grupales.

- Los sistemas y mecanismos de evaluación.

- Las características de los destinatarios: nivel educativo, desempeño profesional, valores y elementos culturales. 
La tecnología educativa profesa una nueva pragmatización del proceso o fenómeno educativo. En la actualidad, se habla en educación, desde conceptos o definiciones con el sentido de lo tecnológico: tecnocracia de la realidad educativa, lenguaje tecnológico, enseñanza programada, enfoque sistémico, etcétera. Hoy, también, hablamos y discutimos sobre los usos de los filmes, la radio, la internet, las redes sociales, las wikis, la televisión, los "video beams", etcétera, todos de incumbencia pedagógica por sus aplicaciones y funcionalidad al proceso educativo.

Existe una modalidad de uso instrumental en lo que a tecnologías educativas respecta y esa es la videoconferencia. El sistema de la videoconferencia es un recurso didáctico altamente valorado en propósitos, objetivos y logros en el proceso moderno de la educación, quizás apologizado con el nacimiento de la red Youtube de internet como recurso didáctico.

Jesús Salinas y Bárbara De Benito (2009), consideran que hoy se puede hablar de tres tipos de videoconferencia: de escritorio, de reuniones y de aula. Respecto a la videoconferencia de escritorio es aquella que permite su difusión y desarrollo mediante el llamado ordenador personal y el uso de la cámara web, sumado al programa que permita la conexión. El tipo denominado videoconferencia de reuniones es de uso recomendado en reuniones, justamente, o seminarios con participación de máximo 8 o 10 personas. Mientras que el tercer tipo, la videoconferencia de aula, busca reproducir la enseñanza presencial, que en este caso el dispositivo tecnológico es mayor.

Para ambos investigadores, la videoconferencia es un instrumento tecnológico usado en la educación con el fin de "romper" con las barreras espaciales (con lo remoto, las distancias físicas), básicamente. La videoconferencia ocupa y necesita, por ende, de un equipamiento formal y básico: la cámara, el micrófono, el sistema de megafonía, la tecnología en cuestión o protocolo de transmisión y el sistema de videoconferencia que digitaliza, codifica y decodifica la imagen, el sonido, los datos y los transmite; agréguese que la videoconferencia también facilita el intercambio de información o compartir aplicaciones (p. 232).

Entre la variedad de aplicaciones educativas de la videoconferencia se hallan: la educación a distancia, conferencias, tutorías, sesiones diversas, encuentros virtuales, entre otras (Salinas y De Benito, 2009, p. 234). Puede, entonces, considerarse que la videoconferencia se enmarca en el rubro de la Enseñanza audiovisual. Esta investigación en cuestión 
se convierte en un intento por visualizar, con mayor claridad, "el problema" docente en la enseñanza de la filosofía mediante el uso de las TIC.

Torres (2008) define al considerar que "[d]eben utilizarse los particulares poderes tecnológicos de los nuevos medios, para estructurar sólidamente los equipos humanos de trabajo de directivos, de profesores, de estudiantes, dando una nueva dimensión real a las posibilidades virtuales que la tecnociencia ha posibilitado"(p. 170) su postura al respecto del asunto de la investigación en general respecto de las TIC.

Él mismo afirma que "es preciso tener presente que la Educación a Distancia es, por antonomasia, una educación de los medios y con los medios" (p. 172). Con esta definición, se declara bien el aspecto esencial de la pedagogía a distancia, a manera de lo substancial en sí misma. Y por supuesto la pertinencia de la videoconferencia en la educación a distancia. En tanto Juan Díaz y Adair Martins (1982, pp. 20-21) concluyen, con respecto a la mejora en la educación superior, con el uso de la tecnología, lo siguiente:

a. Tener una visión integral de los problemas que afectan la enseñanza.

b. Comprender el proceso de aprendizaje.

c. Estimular la participación activa de los alumnos.

d. Mejorar la comunicación entre profesores y alumnos.

e. Desarrollar en los alumnos la actitud y la habilidad requeridas para la investigación.

f. Racionalizar la evaluación.

g. Conocer mejor al alumno como persona y como miembro de una comunidad.

h. Enseñar a los alumnos a estudiar y a aprender.

i. Planear los cursos.

j. Saber cómo introducir innovaciones.

k. Crear en la institución una unidad de apoyo pedagógico.

Todas estas consideraciones en, y para, la planificación de una lección didáctica, incluso filosófica, motivadora y significativa.

\section{Las ventajas y desventajas de la implementación de la videoconferencia}

Valverde (2011) realizó una investigación que le permitiera entresacar resultados y conclusiones que evidenciaran la congruencia 
entre la educación y el uso de las TIC. El objetivo fundamental consistió en interpretar no solo la utilización de las tecnologías educativas, sino además, entrever las consecuencias y situaciones con respecto al advenimiento e implementación de estas tecnologías en estudios de un posgrado de la UNED, en el cual se utilizan tecnologías educativas, incluso las videoconferencias. Una de sus deducciones fue: “...lo que puede desbordar las posibilidades del control que el docente normalmente ha demostrado en el ámbito del aula y que no quisiera perder"( $\mathrm{p}$. 10), o sea, el temor a perder el control por parte del docente por la intervención de estas modalidades instrumentales tecnológicas es real.

Valverde (2011) también acota que es fundamental la labor del docente en tanto "...orientar y guiar al estudiante en la búsqueda de información relevante, a partir de los materiales de apoyo y las opciones tecnológicas" (p.15). En sus conclusiones, además, expone que los docentes "...hacen uso parcial de los recursos tecnológicos para apoyar su labor"(p. 15), contrastando esto, y por su parte, el citado Torres opina que "el educador de hoy debe perderles el miedo a los nuevos medios tecnológicos" (p. 171).

Los ya mencionados Salinas y De Benito (2009, p. 235), citan las que consideran principales estrategias didácticas utilizadas en la docencia a través de videoconferencias:

a. Expositivas: clase magistral, estudio de casos e invitación de expertos.

b. Demostrativas: demostración de objetos y resolución de problemas.

c. Participativas: debates, discusiones, preguntas y respuestas, exposiciones de trabajo.

d. Colaborativas: realización de tareas, trabajo en grupo.

Con Salinas y Benito, entonces, las videoconferencias representan para la educación a distancia: "el sistema por excelencia... cuando profesor y alumno se encuentren alejados geográficamente" (p. 246). De igual manera, Mitra (2012) refiere a comprender que las videoconferencias solucionan en gran medida los dilemas de acceso a ciertas circunstancias educativas, es decir, en donde la dificultad radica en la imposibilidad o dificultad a asistir a una clase presencial, o bien, cuando se carece de infraestructura. La tecnología no es sino un buen acicate para la curiosidad de los alumnos y además para que aprenda a aprender. 
El profesor Burbules (2012) expresa la evidente potencialidad de las tecnologías en la transformación de los entornos educativos actuales. Estas herramientas modernas son eso, instrumentos pedagógicos, pero que han variado en sumo las sociedades escolares y que son aliados al acceso de la información.

Por su parte, Zelaya (2007), considera las siguientes ventajas de las TIC para los docentes: reciclaje y actualización profesional (alfabetización con respecto a las nuevas tecnologías educativas), metodología más dinámica y activa, gran conjunto de recursos didácticos para la enseñanza, investigación didáctica en el proceso enseñanza-aprendizaje y atención a las necesidades y características educativas especiales.

En tanto que como ventajas para los alumnos este autor cita los siguientes: aprendizaje lúdico y atractivo, favorecen a las necesidades especiales educativas, personalización del aprendizaje (el alumno elige los medios que considera más viables), acceso a una gran cantidad de espacios de aprendizaje, posibilidad de gran cantidad de relaciones con otros compañeros (as) y flexibilidad en el proceso de aprendizaje.

\section{Relación docente-discente en ambientes virtuales}

Con las modalidades virtuales, el proceso de comunicación se ve interferido por una nueva manera de llevarlo a cabo; la interacción y el intercambio del conocimiento o aprendizajes se lleva a un nivel distinto, con la posibilidad de la transmisión de este y su entendimiento, pero con otras limitaciones diferentes a las que en una clase presencial podrían darse, por ejemplo, la identificación de la psicología real del ambiente, que siempre media a través de las emociones.

Considérese que estas modalidades de enseñanza-aprendizaje exigen el conocimiento del uso de las tecnologías como tales, de parte de uno y otro actor del fenómeno educativo; definitivamente, la formación educativa de docentes y estudiantes ha de valorar estas nuevas condiciones de actuar en su medio.

La relación entre profesor y alumno en ambientes virtuales se ve inducido al necesario manejo de herramientas y técnicas informáticas, las que se hallan no solo en boga sino que exigen su uso. La conveniencia o inconveniencia de las nuevas tecnologías educativas virtuales y su influencia en las relaciones docente-discente quizás ya se pueden mostrar en sus efectos; sin embargo, estas nuevas realidades en el campo de 
lo educativo han de asumirse. En cuanto al "nuevo papel" del docente en los ambientes virtuales por ende en la historia de las tecnologías educativas modernas, Zelaya (2007) expone la importante definición:

Las funciones tradicionales del docente cambian radicalmente cuando se desarrollan actividades en un entorno virtual de educación a distancia, basado en la eliminación de las limitaciones normales, tales como espacios geográficos, presencia física y otras de tipo temporales, permitiendo ahora crear grupos más heterogéneos, dispersos geográficamente y muchas veces multiculturales y de diversos grupos socioeconómicos. (p. 25).

Zelaya (2007) también asume, respecto de la respuesta positiva o negativa de los docentes frente a la casi imperativa utilización de las nuevas tecnologías educativas (TIC), que sea cual sea esta su actitud, estará condicionada por aspectos como los siguientes:

- La infraestructura de comunicaciones de que disponga.

- $\quad$ El espacio disponible en su centro habitual de trabajo que permita la fácil integración de la tecnología.

- Su preparación para el uso de esta tecnología (tanto desde el punto de vista de hardware como del software).

- La disponibilidad de docentes para una formación permanente que le garantice no perder la "carrera tecnológica".

Zelaya (2007) plantea varios roles y funciones que los docentes actualmente deben asumir debido a estos ambientes tecnológicos, él cita que los docentes de estas nuevas coyunturas tecnológicas en educación han de ser consultores de la información (buscan información y recursos que le den soporte a su docencia), colaboradores en grupo, trabajadores solitarios (en caso de teletrabajo o teleformación), facilitadores del aprendizaje, desarrolladores de cursos y materiales, y hasta de supervisores académicos de sus alumnos.

Zelaya (2007) considera que existen ciertos elementos de la personalidad del alumno que facilitarían su inserción y adaptación a las modalidades de educación a distancia: la automotivación, la capacidad de aprendizaje (aprendizaje significativo, autogestivo y autónomo), la disciplina y el orden, la capacidad de investigación y la auto-evaluación por citar. 
De esta manera, la videoconferencia, como medio didáctico requiere de un planeamiento adecuado a los intereses actuales de los estudiantes, con tal de motivar el aprendizaje y de parte del docente un compromiso inherente a su vocación para que por medio de la videoconferencia se logre mejores resultados didácticos.

\section{El marco metodológico de la investigación}

La investigación se dio acorde con el paradigma postpositivista (el papel del sujeto toma relevancia en cuanto considera valioso el entorno de creencias, valores, intereses y demás, que influyen en el fenómeno estudiado), su pregunta ontológica es de carácter realista-crítica, ya que la epistemología se denota objetivista modificada, pues hay incumbencia de análisis e interpretación.

La investigación es de carácter descriptivo, al buscar describir no solo al fenómeno como tal, sino que a partir de la información conseguida mostrar las características de la comunidad elegida, y su perspectiva respecto al uso e implementación de las videoconferencias, en este caso, para posteriormente evaluar los datos recolectados. El enfoque del estudio es de orden mixto.

La muestra de sujetos de la investigación fue la siguiente: docentes de Filosofía de la Cátedra de Filosofía, una muestra de estudiantes de cursos de Estudios Generales de la UNED, personal encargado de la sección de videoconferencias de la Universidad Estatal a Distancia (coordinador de la Sede Central, coordinadores de centros universitarios y la Productora de Videoconferencias de la UNED) y el encargado de la Cátedra de Filosofía de la UNED. Se contó al final con la participación de 13 docentes (tutores) de un total de 21 y de 65 estudiantes durante el primer cuatrimestre del año 2013.

Para la recolección de la información, se aplicaron entrevistas estructuradas y no estructuradas, así como encuestas y cuestionarios. El análisis de datos se llevó a cabo mediante la triangulación de datos, obtenidos a partir de la variedad de fuentes de información que participaron. A partir de la implementación de los instrumentos de recolección de la información se procedió a realizar:

1. Análisis de datos partiendo de la información recibida de parte de los actores indagados. Con esta información, se realizó un 
cotejo cuantitativo correspondiente, en sus casos de pertinencia de análisis de estas calidades. En otros casos, con índoles cualitativas, tales como las entrevistas al catedrático y a encargados de las oficinas de videoconferencias. Y así se realizó un análisis de hallazgos que le dieran a la investigación y al investigador una perspectiva fiable para elaborar la interpretación de los datos recabados, la exposición de los resultados, de las conclusiones y las recomendaciones generales.

2. Un análisis de los datos proveídos por tutores, estudiantes, catedrático y especialistas en videoconferencias de la Universidad Estatal a Distancia.

3. La organización de la información recabada permitió encontrar similitudes, diferencias, relaciones, patrones y significados entre datos que a la vez permitió generar interpretaciones.

4. Incluso se acudió a un análisis y síntesis de lo que la sección de videoconferencias de la UNED presenta en su plataforma de servicios específicamente en los cursos de filosofía para los Estudios Generales.

\section{Los hallazgos de la investigación}

Respecto de los hallazgos logrados a través de la investigación se muestra desde cada objetivo específico:

Del Objetivo 1: Analizar las videoconferencias como medio de aprovechamiento pedagógico en el campo de la Filosofía.

El encargado de la Cátedra de Filosofía estimó que, respecto de las videoconferencias y su aprovechamiento pedagógico en el campo de la Filosofía, se les da alta relevancia en el sistema educativo de la UNED, y en la Cátedra de Filosofía, en tanto, sus cursos ofrecidos a los estudiantes de Estudios Generales. Sin embargo, afirma que se necesita de más desarrollo y ampliación de alcance, posicionamiento espacio-temporal.

Los encargados de departamentos de videoconferencias de la UNED, en centros universitarios, indican que las videoconferencias resultan convenientes para los docentes y estudiantes de la UNED, pues se acortan las limitantes físicas y de desplazamiento. Pero deben ser 
implementadas en todas las asignaturas ofrecidas por la Universidad Estatal a Distancia. Este recurso tecnológico resulta ser un complemento oportuno y válido para los estudiantes y de la tutoría presencial.

El Coordinador del Programa de Videoconferencias y la Productora de Videoconferencias de la UNED indican lo siguiente (que lo generalizan a todas y cualquiera de las asignaturas del curriculum académico universitario):

1. Los estudiantes no solo por el acceso a las videoconferencias logran el provecho de esta herramienta tecnológica y virtual, sino por medio de otras modalidades de las cuales pueden lograr nuevos y mayores resultados, por ejemplo, con videoconferencias móviles, vídeo tutorías o vídeo talleres.

2. Las videoconferencias son excelentes medios educativos mediadores de la socialización del conocimiento (por ejemplo, por medio de los chats).

3. Sí se brindan capacitaciones a tutores de la Universidad (UNED) en cuanto a la implementación de las videoconferencias.

4. Las videoconferencias son oportunas y de valor pedagógico en tanto "aterricen" en la realidad, por ejemplo, con el uso de la metáfora.

5. La videoconferencia tomará mayor posicionamiento didáctico, en tanto, no sea más concebida como apoyo didáctico, sino como momento oportuno de interacción educativa.

6. Una videoconferencia en la UNED puede "vivirse" sea presencialmente (apersonarse en la Universidad y por ende en la sala de videoconferencias), desde el hogar, en las salas de videoconferencias de algunos centros universitarios, o mediante el blog por instancias "streaming" (transmisión de audio y video por medio de la red internet).

Por su parte, los tutores de filosofía y los estudiantes indagados proporcionaron los siguientes datos e información, los cuales se muestran con figuras: 
Figura 1. Valoración de la videoconferencia por tutores y estudiantes

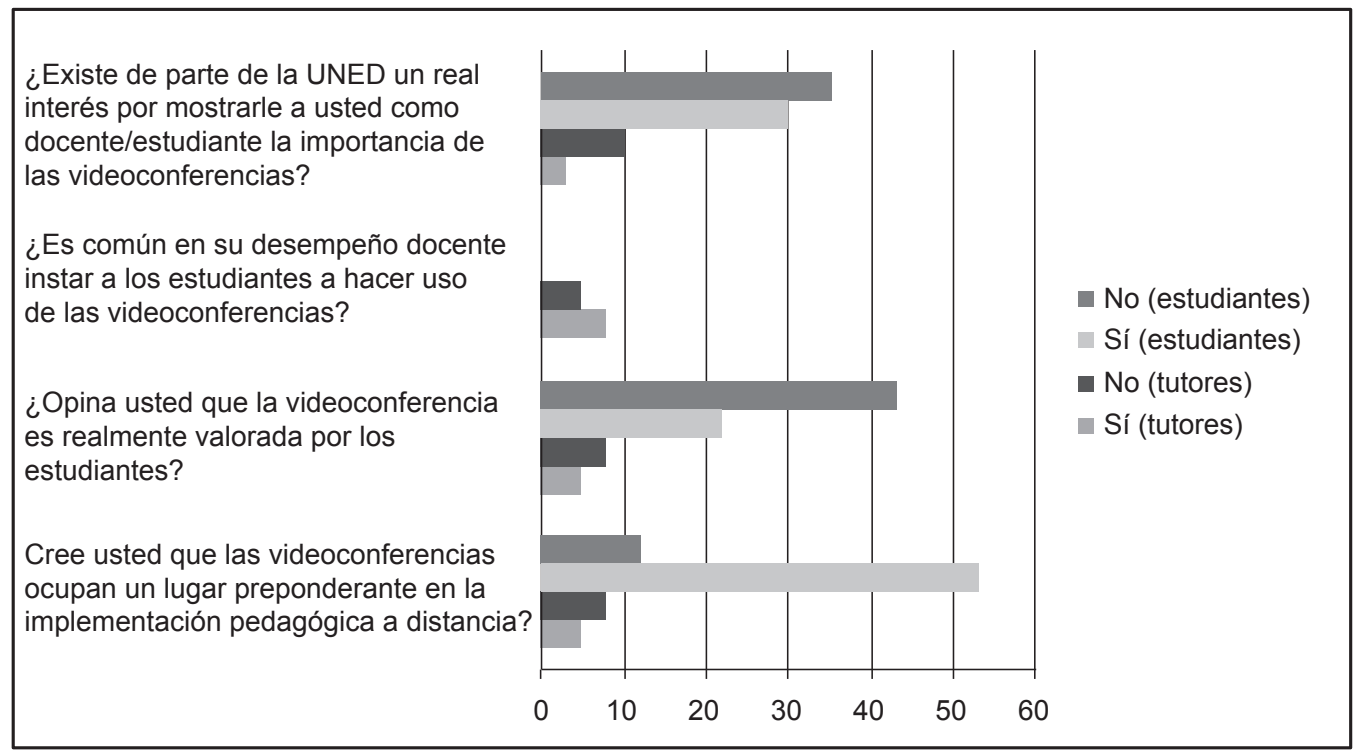

Fuente: Instrumento C y D: Cuestionarios dirigidos a los docentes-tutores de la Cátedra de Filosofía de la UNED, y a los estudiantes.

Ocho de trece tutores consideran que las videoconferencias no ocupan un lugar preponderante en la implementación pedagógica a distancia, cinco tutores consideran que sí lo ocupa. Cincuenta y tres estudiantes consideran que sí lo son y veintitrés que no lo son. Ocho tutores consideran que las videoconferencias no son valoradas realmente por los estudiantes, cinco tutores consideran que sí lo son. Veintidós estudiantes aducen que sí son valoradas, pero cuarenta y tres que no lo son. Ocho tutores indican que no instan a los estudiantes a hacer uso de las videoconferencias, cinco sí indican instar a ello. Diez tutores consideran que la UNED no les inculca la importancia del uso de la videoconferencia, tres que sí. Treinta estudiantes estiman que sí lo hace, treinta y cinco que no.

Consecuentemente, se aprecia que los tutores tienen cierto recelo del uso de la videoconferencia, aunque de parte de los estudiantes se muestra mayor apertura. En cuanto a la capacitación que se ofrece, y respecto al uso de las videoconferencias como medio didáctico, se presenta lo siguiente: 


\section{Figura 2. La videoconferencia en la filosofía y capacitaciones} en uso de la videoconferencia.

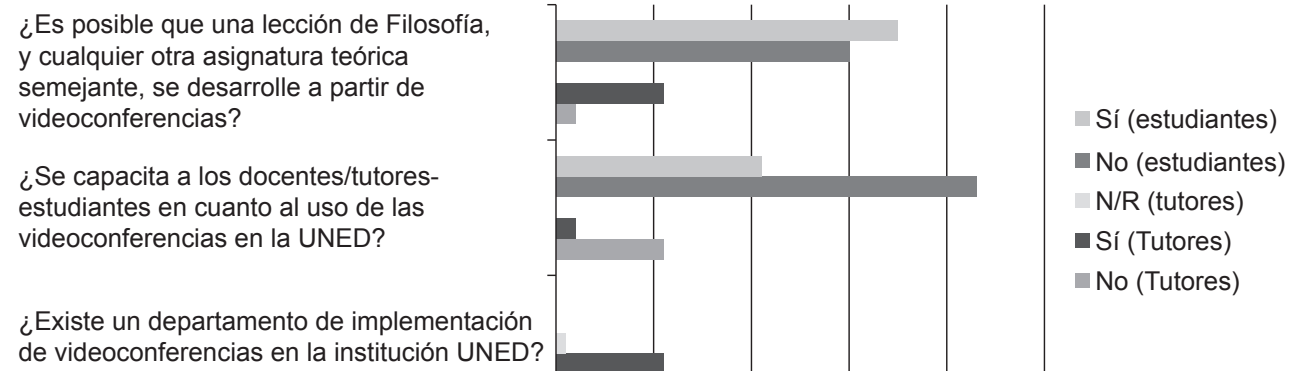

Sí (estudiantes)

No (estudiantes)

N/R (tutores)

- Sí (Tutores)

No (Tutores)

Fuente: Instrumento C y D: Cuestionarios dirigidos a los docentes-tutores de la Cátedra de Filosofía de la UNED, y a los estudiantes.

Once tutores conocen de la existencia de departamentos de videoconferencias de la UNED, uno no lo conoce y uno no responde. Dos tutores aceptan que existe la capacitación de la UNED en cuanto el uso de las videoconferencias, once tutores no reconocen tales capacitaciones. Cuarenta y tres estudiantes asumen que no lo hace (capacitarse) y veintiuno que sí lo hace. Dos tutores consideran que no es viable elaborar una clase de filosofía o de otra asignatura semejante, mediante las videoconferencias, once tutores consideran que sí. Mientras que treinta y cinco estudiantes consideran que sí es posible y treinta que no lo es. La siguiente fue otra consulta:

Figura 3. Asiduidad del estudiante a la videoconferencia.
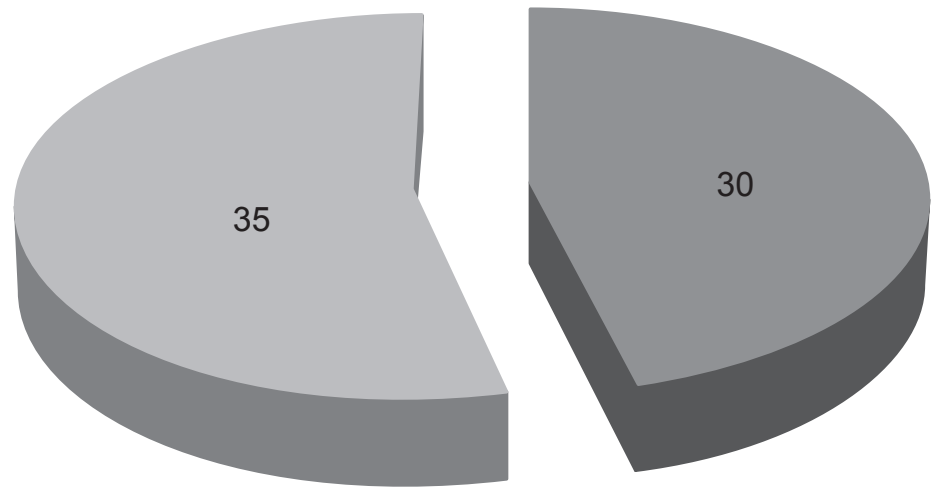

- Sí

No

Fuente: Instrumento D: Cuestionario a estudiantes universitarios de la UNED, curso de Filosofía, Estudios Generales. 
Consideramos que casi la mitad de los estudiantes afirma su asiduidad a las videoconferencias. En otra consulta, el resultado fue el siguiente:

Figura 4. Consideración de la importancia de la videoconferencia por parte del estudiante.

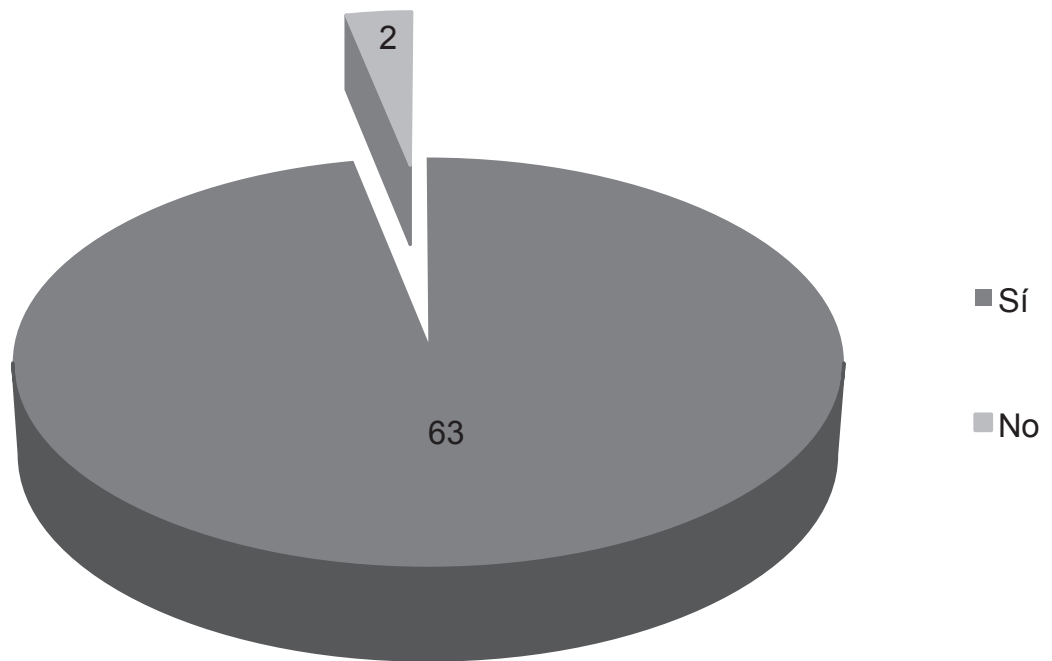

Fuente: Instrumento D: Cuestionario a estudiantes universitarios de la UNED, curso de Filosofía, Estudios Generales.

Es realmente estimable el hecho de que 63 estudiantes de 65 han considerado importante el uso de la videoconferencia en la educación a distancia. Aspecto que se contrasta con la información aportada por los estudiantes en la Figura 1.

Del Objetivo 2: Explorar la funcionalidad de las videoconferencias en el campo pedagógico.

El encargado de la Cátedra de Filosofía estima que, respecto de las videoconferencias y su funcionalidad pedagógica, a manera de consulta son muy factibles, pero se daría esta funcionalidad si existiera realmente relacionabilidad docente-estudiante. Son muy funcionales en debates y producción de conocimiento, pero la videoconferencia no podrá sustituir a la clase presencial.

Los encargados de departamentos de videoconferencias de la UNED, centros universitarios, relatan los siguientes comentarios: 
1. La UNED cuatrimestralmente implementa gran cantidad de ellas, incluso en vivo (señal streaming) con posibilidad de chat.

2. Las videoconferencias se suben a la videoteca para su acceso.

3. En materias como la filosofía no son muy convenientes pues es una materia de sumo análisis y el estudiante tiende a cansarse. Por eso, han de incluirse actividades interactivas.

El coordinador del Programa de Videoconferencias y la productora de Videoconferencias de la UNED indican lo siguiente, pero lo generalizan a todas y cualquiera de las asignaturas del curriculum académico universitario:

1. Las videoconferencias en educación son funcionales en tanto se dinamicen en su presentación por el docente, quizás mediante actividades lúdicas.

2. Son funcionales en tanto se visualicen las poblaciones a quienes se han de dirigir: tipo de estudiantes según su nivel académico o regional en el país.

3. Son funcionales si se organizan y planifican esmeradamente de parte de los actores inmediatos que han de transmitir el conocimiento.

4. Se convierte en una necesidad, un diseño curricular que incluya cursos en línea, audiovisuales, multimedia, etcétera.

5. La funcionalidad de la videoconferencia será más latente y patente en cuanto el tutor "videoconferencista" tenga conocimientos pedagógicos y tecnológicos.

6. Es sumamente importante la inducción y capacitación tanto a docentes tutores como a estudiantes, no solo del uso de la videoconferencia, sino además del conocimiento de su pertinencia en la formación académica.

7. Es fundamental que la videoconferencia lleve a prácticas inmediatas o análisis de realidades actuales para que esta sea aprovechada de mejor manera.

8. La videoconferencia cumplirá con sus propósitos educativos si se consideran aspectos de carácter andragógico, circunstanciales, contextuales y de perspectivas. Generando diálogo, razonamiento, análisis y reflexión.

9. La posibilidad de "traer" invitados a las videoconferencias, de parte de los tutores "videoconferencistas" dinamizan más la actividad. 
Por su parte, los tutores de Filosofía proporcionan los siguientes datos e información a la investigación, conforme estima el objetivo del caso:

1. Dos tutores consideran que la videoconferencia no es funcional en educación a distancia para la realización de una clase de filosofía, once lo consideran que sí.

2. Trece tutores consideran que la videoconferencia sí es funcional en sistemas educativos tradicionales (no a distancia), ninguno considera lo contrario.

Acá se reconviene en que la mayoría de los tutores indagados sí consideran oportuno el uso de la videoconferencia como medio didáctico en y para cursos filosóficos. Incluso para sistemas de educación tradicional. Puede considerarse que existe algún desconocimiento de parte de los tutores acerca de lo que la videoconferencia es, en su naturaleza y aplicación educativa.

Del Objetivo 3: Conocer desde los diferentes modelos teóricos, las ventajas y desventajas de la implementación de la "videoconferencia" en el área de la filosofía.

El encargado de la Cátedra de Filosofía estima que:

Las ventajas son:

1. Se evita el desplazamiento espacial.

2. Se permite flexibilidad en la comunicación, especialmente, en la posibilidad de la comunicación sincrónica y asincrónica.

Las desventajas son:

1. Las limitaciones en cuanto a la imposibilidad de la conversación didáctica.

2. Se requiere de la tecnología requerida para lograr los resultados deseados, en algunas situaciones, no se cuenta con la tecnología.

Los encargados de Departamentos de Videoconferencias de la UNED, Centros Universitarios, relatan los siguientes comentarios acerca de lo solicitado: 
Las ventajas son:

1. Se facilita el proceso de aprendizaje del estudiante ya que le permite recibir una clase sin necesidad de trasladarse a un lugar en específico.

2. Se rompen distancias y permiten que personas de diferentes lugares compartan una clase en un mismo momento.

3. Le permite al profesor atender más estudiantes en una sola clase.

4. Es todo un medio más para que el estudiante evacue sus dudas.

5. Se puede permitir el compartir ideas entre los actores educativos.

6. Posibilita realizar talleres y capacitaciones en diferentes áreas.

Las desventajas son:

1. Un gran dilema es cuando el sistema "se cae", por lo tanto, no hay transmisión de la videoconferencia o clase.

2. La ausencia de fluido eléctrico obstaculiza el funcionamiento del sistema.

3. En ocasiones se pierde el contacto personal entre los asistentes, cuando no hay interacción entre los participantes.

El Coordinador del Programa de Videoconferencias y la Productora de Videoconferencias de la UNED indicaron lo siguiente, pero lo generalizan a la filosofía y a todas, y cualquiera, de las asignaturas del curriculum académico universitario:

Las ventajas son:

1. Se permite la participación de los estudiantes.

2. La videoconferencia genera un cambio en la concepción de la educación, lo que no ha de implicar que la tutoría presencial se debe eliminar.

3. Se adquieren nuevas competencias y conocimientos en la entrega del conocimiento y su posibilidad formativa.

4. Posibilidades de innovar y creatividad en la educación y su proceso.

5. Posibilidad de que desde las cátedras se formen a "videoconferencistas" y que estos formen a otros más. 
Las desventajas son:

1. La falta de orden y estimación de la videoconferencia de parte del tutor, cátedras e inclusive Escuelas de la Universidad.

2. No se considera el tipo de estudiante al que la tutoría se dirige.

3. La dificultad de creatividad de parte del tutor.

4. El mero cumplimiento por parte de tutores, quienes no se preparan en investigación sino que se abocan al solo texto y su recitación.

5. No media la búsqueda, por parte de las cátedras, de la persona idónea en capacitación y disposición, para que elabore y presente una videoconferencia.

6. Desdén a las capacitaciones en videoconferencias, de parte de cátedras y tutores, y que la Coordinación de Videoconferencias les brinda.

7. La escasa cultura universitaria de la implementación de la videoconferencia.

8. Desconocimiento del instrumento videoconferencia y su metodología en el campo de la educación.

9. Miedos políticos y existenciales en las cátedras y en los tutores para asumir las bondades de las videoconferencias.

Así se determinó una contrastación entre la teoría, expuesta anteriormente y los resultados de la investigación, la cual se detalla a continuación.

1. La modalidad de educación a distancia está consolidada en el país; la UNED representa la institución de educación superior pionera en ello y tiende a fortalecerse por las situaciones propias de la época, en donde muchas personas necesitan trabajar y una posible asistencia a un aula presencialmente se les hace difícil por esa razón.

2. La educación a distancia, no solo permite "reducir" los espacios geográficos y temporales en educación, sino que además cuenta con los medios e instrumentos de punta para proveer en los estudiantes y docentes la consecución de los objetivos didácticos propios a esta modalidad educativa.

3. La tecnología educativa se define como primordial en la UNED y su desempeño educacional, la UNED posee de un amplio sistema organizado de oficinas de videoconferencias, las que facilitan la 
aprehensión del conocimiento mediante éstas. No obstante, estudiantes y tutores indican que la UNED debe realizar mayores esfuerzos por fomentar el uso de la videoconferencia.

4. Se puede considerar funcional el uso de la videoconferencia en la UNED; sin embargo, en cuanto a la realización de una videoconferencia de filosofía surgen ciertas distenciones, especialmente, por el desconocimiento de su uso y efectividad, así como de la poca cultura al respecto.

5. La videoconferencia parece ser de buen provecho para los estudiantes, aunque los tutores de filosofía parecen desdeñar de ella.

6. La videoconferencia muestra como principal ventaja "el rompimiento" espacio-temporal para el aprendizaje y la inclusión de la socialización en ambientes virtuales mediante este instrumento o medio didáctico. Como desventaja, sin embargo, se manifiesta que puede convertirse en un sistema o medio didáctico hermético y poco motivante, ya en la práctica pedagógica.

7. El papel del docente en ambientes de virtualidad es consecuente con las nuevas formas de educar a partir de las tecnologías educativas, pero existen ciertas reticencias a asumir las tecnologías educativas, especialmente, porque aún se considera necesaria la interrelación entre los actores principales del acto educativo.

8. El discente ha de ser capacitado, tanto como el tutor-docente, en el uso y aplicación didáctica de las videoconferencias. Pero este, necesita de una motivación fundamental para que entrevea la riqueza de la videoconferencia.

\section{Conclusiones de la investigación}

Estas son las conclusiones que surgieron de la investigación y del análisis de datos emanados por los participantes.

En cuanto al primer objetivo: "Analizar las videoconferencias como medio de aprovechamiento pedagógico en el campo de la Filosofía, y en la educación superior" se concluye que:

- La videoconferencia resulta ser un instrumento pedagógico o didáctico, considerable en tanto su bienestar con el proceso educativo, es funcional y factible especialmente en la generación de debates. 
- $\quad$ Este recurso didáctico merece de una elaboración más exhaustiva y perfeccionada para que logre mayores alcances y resultados.

- $\quad$ Es necesario un mayor esmero y compromiso por parte de los tutores que presentan las videoconferencias, principalmente, en su confección.

- También es considerable y necesario que el tutor designado para la elaboración y presentación de la videoconferencia, tenga conocimientos de pedagogía y de tecnología educativa.

- Se insiste en la necesaria capacitación de los tutores en cuanto al uso de las videoconferencias.

- El coordinador y la productora apoyan la conclusión anterior agregando la posibilidad de actividades lúdicas y de contextualización de los contenidos para que la videoconferencia se muestre atractiva.

- $\quad$ Son necesarias, consecuentemente, propuestas creativas e innovadores de parte de los tutores.

- Que los tutores no solamente se ocupen de la preparación de una tutoría por el mero cumplimiento de un deber. El desdén va más allá, hasta el poco interés o valoración desde las escuelas y cátedras de la Universidad a la técnica de la videoconferencia.

Los tutores de la Cátedra y estudiantes denotan las siguientes conclusiones:

- $\quad$ Respecto de la videoconferencia y su uso, un marcado porcentaje, más del $50 \%$ de ellos, no consideran pertinente la implementación de la videoconferencia en la educación a distancia. Por su parte, los estudiantes consideran lo contrario, más del 50\% sí la consideran pertinente.

- La mayoría de los tutores-docentes no estimulan el uso de la videoconferencia entre sus estudiantes. Pero, un porcentaje similar al que adujo lo anterior, estiman que la universidad tampoco les inculca la importancia del uso de las videoconferencias.

- La muestra de estudiantes "se parte" en "cincuenta-cincuenta" respecto de si la Universidad les inculca el uso de las videoconferencias.

- Prácticamente, todos los tutores conocen de la existencia de los departamentos institucionales de videoconferencias, pero la mayoría no sabe de la realidad de capacitaciones en el uso de éstas. En los estudiantes, más del 50\% no conocen de capacitaciones para ellos. 
Respecto al segundo objetivo que indica: "Explorar la funcionalidad de las videoconferencias en el campo pedagógico y andragógico universitario" el Coordinador del Programa de Videoconferencias, la Productora de Videoconferencias y algunos encargados de los Departamentos de videoconferencias de la UNED dicen:

- La videoconferencia sí es funcional en tanto se reducen las limitaciones espaciales y de desplazamiento, evento objetivo didáctico en la naturaleza de la modalidad de la educación a distancia. Las denotan viables para todas las asignaturas, pero a manera de complemento didáctico, pues lo facilitan y motivan, aunque el coordinador y la productora estiman que las videoconferencias no son en realidad apoyos educativos, sino "momentos de interacción educativa".

- $\quad$ El coordinador y la productora explican que las videoconferencias son medios de socialización del conocimiento.

- $\quad$ Poco menos del 50\% de los estudiantes son asiduos al uso de la videoconferencia, pero casi la totalidad de estos sí la consideran importante en la educación a distancia.

- $\quad$ Para la investigación, hubo algunos problemas para acceder a cierta información considerada de suma utilidad para el desarrollo de esta investigación y sus resultados, por ejemplo, la imposibilidad de acceder al orden protocolario y de ejecución de una capacitación en videoconferencias. El investigador se sumó a un grupo de tutores que recibirían una capacitación en el tema de videoconferencias, pero tal capacitación no se dio pues ese día jugaba la selección de fútbol.

En lo que se refiere al tercer objetivo: "Conocer desde los diferentes modelos teóricos, las ventajas y desventajas de la implementación de la videoconferencia en el área de la filosofía en la educación superior", se concluye lo siguiente:

- $\quad$ Se considera la pertinencia de la clase presencial

- La videoconferencia en materias como la filosofía puede guardar mayor dificultad de elaboración y aprovechamiento, pues son asignaturas/materias de sumo análisis, se recomienda la interacción.

- $\quad$ El coordinador y la productora sí consideran que materias curriculares como la filosofía son viables mediante el uso de la 
videoconferencia si hay aprecio a la técnica y preparación anticipada y meditada.

- Los estudiantes en un 50\% considera que es viable la clase de filosofía mediante la videoconferencia. Los tutores consideran que la videoconferencia es funcional más en sistemas tradicionales.

- Respecto de la viabilidad de la videoconferencia para la elaboración de una tutoría de filosofía, dos tutores consideran que no lo es, $90 \%$ considera que sí es, aunque ya más del $50 \%$ habían expuesto que la videoconferencia no es pertinente en la educación a distancia.

Respecto al cuarto objetivo: "Determinar una propuesta pedagógica que posibilite la aprehensión y consecuente valoración de la videoconferencia como medio didáctico entre los estudiantes universitarios de la UNED".

Se presentó la propuesta para esta investigación con interés didáctico dirigido a docentes, tutores y estudiantes del sistema de educación a distancia en la UNED y otras universidades. También se presentó al docente-tutor de la enseñanza de temas filosóficos y cualquier docente de la educación universitaria y de los diversos campos del saber.

El objetivo general es el siguiente:

Diseñar un conjunto de talleres educativos que implementen el conocimiento, valoración y práctica de las videoconferencias en Centros Universitarios de la UNED, particularmente en aquellos que no posean departamentos de videoconferencias propios.

Los objetivos específicos son:

1. Elaborar una serie de talleres, con incumbencia en la didáctica de la videoconferencia, para propiciar docentes, administradores y estudiantes en cualquier saber, dispuestos a la comprensión y uso de esta.

2. Implementar esos talleres con la participación de profesionales en el campo de las videoconferencias, y quienes han de estar dispuestos a visitar tales centros universitarios elegidos, en conjunto con la Coordinación de Extensión Universitaria.

3. Evaluar los resultados de tales talleres con el fin de concientizar de la relevancia del aprendizaje del uso de las videoconferencias.

Esta estrategia de enseñanza y aprendizaje se ha denominado: Talleres educativos para docentes y estudiantes de la UNED en el uso 
de las Videoconferencias. Estos talleres tratarían de solventar algunas de esas carencias académicas identificadas, propiciarían investigación educativa y posibilitarían el "aprender a aprender", desde el pensar, cooperar, comunicar, empatizar y criticar, que facilitaría la autonomía educativa y el uso de tecnologías educativas.

\section{Referencias bibliográficas}

Burbules (2012). Videoconferencias Dr Burbules: Aprender en todo momento y lugar (Parte 3-9). Recuperado de http://www.youtube.com/watch?v=ST1bc5B86Y4

Díaz, J et al. (1982). Estrategias de enseñanza aprendizaje: Orientaciones didácticas para la docencia universitaria. San José, costa Rica. IICA.

Mitra, S. (2012). Sugata Mitra: Los niños pueden aprender por símismos. Recuperado de http://www.youtube.com/watch?v=-u2LX-ZKs5I

Ruíz, S. (2009). Educación a distancia: Apuntes sobre sus orígenes y justiprecio como recurso práctico y poderoso para la educación permanente a la luz del cambio intrageneracional. Ensayos Pedagógicos: Umbral del Conocimiento, 71-86.

Salinas, J. et al. (2009). Tecnologías para la educación: Diseño, producción y evaluación de medios para la formación docente. Madrid, España: Alianza Editorial.

Torres, R. (2008). Los nuevos paradigmas en la actual revolución científica y tecnológica. San José, Costa Rica: EUNED.

Valverde, J. (I Semestre Setiembre, 2011). En Rev: UMBRAL. La tecnología en el proceso educativo de un posgrado del área educativa de la UNED. No XXVIII, 10-20.

Zelaya, J. (2007), Educación a Distancia: Una Alternativa para los Sistemas Educativos. San José, Costa Rica: Colección IDER. 